\title{
INFLUENCE OF LUMBOPELVIC STABILITY ON DEADLIFT PERFORMANCE IN COMPETITIVE POWERLIFTERS
}

\author{
Naoki Sakakibara ${ }^{1}$, Sohee Shin ${ }^{1}$, Tsuneo Watanabe ${ }^{1}$, and Toshio Matsuoka ${ }^{1}$ \\ ${ }^{1}$ Department of Sports Medicine and Sports Science, \\ Gifu University Schools of Medicine, Japan
}

\section{SUMMARY}

The deadlift exposes the spine to extreme loads and requires adequate lumbopelvic (core) stability. Deadlift performance may be influenced by the neuromuscular control of the trunk. In this study, we aimed to compare the transversus abdominis contractile rates in an elite powerlifter with those of a control group during deadlift and estimate the relationships between core stability and deadlift performance. In the present controlled laboratory study, 16 powerlifters [ 8 male national-level powerlifters and 8 male regional-level powerlifters (control group)] were tested for changes in transversus abdominis thickness to evaluate transversus abdominis contractility at each deadlift phase using ultrasound imaging. Compared with the control group, the elite powerlifters showed a higher transversus abdominis contractile rate when the weight was at knee level $(2.16$ vs. $1.74 ; p=.04)$. There were no significant differences between the transversus abdominis contractile rates in both groups when the weight was at the floor and top level. This study reveals that deadlift performance may be influenced by transversus abdominis contractility (lumbopelvic stability).

Key Words: athletic performance, core stability, lumbopelvic stability, transversus abdominis.

\section{INTRODUCTION}

Competitive powerlifting requires explosive muscular power and exposes the spinal column to extreme forces. In this sport, the following three lifts are included: squats, bench press, and deadlift. Each lift has its own primary movers, with the deep trunk muscles acting primarily as stabilizers. The deadlift was chosen for this study because this lift exposes the spine to extreme loads and requires adequate lumbopelvic stability. In the deadlift, a powerlifter lifts the barbell off the floor and stands in an upright position. The lift concludes with the powerlifter standings with the knees and hips extended and the scapula retracted. Previous studies indicate that the deadlift includes the following three phases of movement (Hales, Johnson, \& Johnson, 2009; McGuigan \& Wilson, 1996): first when the powerlifter applies force to the bar to lift it off the floor; second when the bar passes the knee; and third when the powerlifter lifts the bar into a full upright position.
The core muscles do not generate power but the thigh, gluteal, and back muscles are considered the primary power generators that maintain core stability (McGill, 2010). Core stability is related to the body's ability to control the lumbopelvic region in response to internal or external disturbances; it is a foundation by means of which the trunk produces, transfers, and controls force, enabling motion of terminal segments in the kinetic chain (Cook, 2001; Kibler, Press, \& Sciascia, 2006; Liemohn, Baumgartner, \& Gagnon, 2005; Panjabi, 1992). The core has been defined as a double-walled cylinder with the diaphragm as the roof, abdominals as the anterior wall, paraspinals and gluteals as the posterior wall, and pelvic floor and hip musculature as the bottom. Coordinated activation of these muscles is vital for core stabilization (Bergmark, 1989; Panjabi, 1992). The core has received particular attention because it serves as the center of the functional kinetic chain. The ideal core stability includes the ability to control the lumbopelvic region and transfer the greatest torque levels to the distal 
segments (McGill, 2002). Therefore, core instability is believed to cause alterations in energy transfer, resulting in a reduction in athletic performance. Consequently, the ability to stabilize the lumbopelvic region can significantly affect the deadlift performance by maintaining the kinetic chain. Thus, deficits in core stability may lead to an abnormal kinetic chain, which in turn may influence the deadlift performance.

Transversus abdominis ( $\operatorname{Tr} A)$ is considered as a primary stabilizing muscle in the lumbopelvic area (Cresswell, Oddsson, \& Thorstensson, 1994; Hodges, 1994; Maughan, Watson, \& Weir, 1984; Miller \& Medeiros, 1987). TrA originates from the iliac crest, the lower six ribs, lateral raphe of the thoracolumbar fascia, and then passes medially to the linea alba. Because of its transverse fiber orientation, the $\operatorname{Tr} A$ controls the lumbar spinal posture by increasing the tension in the thoracolumbar fascia and intra-abdominal pressure (Cresswell, Grundstrom, \& Thorstensson, 1992; Cresswell et al., 1994; Oddsson, 1990). Springer and Gill (2007) also suggested that $\operatorname{Tr} A$ is more responsible for motor control than for strength.

A number of studies regarding $\operatorname{Tr} A$ have focused on motor control and sequencing of muscle contractions (Hodges, 1994; Hodges \& Richardson, 1996; Hodges, Richardson, \& Hasan, 1997; Misuri, Colagrande, \& Gorini, 1997). In this respect, TrA has a distinct function during movements that involve the trunk (Hodges, 1994; Hodges \& Richardson, 1997, 1998; Hodges et al., 1997). In particular, it has been demonstrated that core stability has a positive influence on athletic performance (Parkhouse \& Ball, 2011). However, no study has evaluated the lumbopelvic stability and its influence on the deadlift performance in competitive powerlifters. To the best of our knowledge, this study is the first to evaluate changes in $\operatorname{Tr} A$ thickness in competitive powerlifters by ultrasound (US) imaging.

Because core stability is considered as one of the factors influencing strength or power output, we hypothesized that a relationship exists between core stability and deadlift performance in competitive powerlifters. Therefore, to investigate the influence of lumbopelvic stability on deadlift performance, we compared TrA muscle contractile rates between national and regional level powerlifters while they performed the deadlift.

\section{METHODS}

\section{Participants}

Sixteen male Japanese powerlifters recruited from a local powerlifting gym participated in this study (8 national level and 8 regional level male powerlifters). All participants completed a questionnaire to assess their suitability for the study. The following were the exclusion criteria: low back pain in the past three months, significant spinal deformity, current urinary tract infection, history of surgery in the lumbopelvic area, or history of neuromuscular disease. The participant's physical characteristics were measured by standard procedures (Table 1). The study was explained to the participants before they provided written informed consent.

Several methods have been developed to counterbalance a competitor's bodyweight during powerlifting in order to allow for comparisons among different classes of weight. The Wilks equation, by the International Powerlifting Federation, was used to evaluate the powerlifters' yield, regardless of the body mass. Using this method, the amount of weight lifted by the powerlifter was multiplied by an index based on the body mass. In this study, participants who have participated in previous world championships (2010, 2011, 2012) were categorized as national level (elite) powerlifters and who have never been qualified were categorized as regional level (control) powerlifters.

\section{Procedure}

A number of studies used needle electromyography to measure TrA contractility (Hodges \& Richardson, 1997; Hodges \& Richardson, 1998). However, this method is invasive and uncomfortable for the participants. Thus, researchers have used US imaging to evaluate the muscle contractility by demonstrating that changes in $\operatorname{Tr} \mathrm{A}$ thickness correlate with changes in the muscle activity (Hales et al.. 2009). Therefore, the muscle thickness can be used to estimate the muscle activity because muscle force correlates with the muscle's cross-sectional area (Kanehisa, Ikegawa, \& Fukunaga, 1994; Maughan et al., 1984; Misuri et al., 1997). US imaging has been used reliably and successfully to measure the lateral abdominal muscles (Bunce, Moore, \& Hough, 2002). In the present study, we conducted US imaging to measure TrA thickness using a US scanner (UF-4500; Fukuda Denshi, Tokyo, Japan) in brightness mode with a $12-\mathrm{MHz}$ linear array transducer. An electroconductive water-based transducer gel was applied to the skin prior to US imaging. The transducer was positioned perpendicular to the abdominal wall, superior to the left iliac crest, and along the mid-axillary line. This placement enables accurate readings and provides optimal image clarity (Misuri et al., 1997). Care was taken to place the transducer in the same spot during data collection. The transducer was placed such that the hyperecho- 
ic interface between the $\operatorname{Tr} A$ and thoracolumbar fascia appeared at the far right of the image, and the transducer's angle was adjusted to optimize image visualization. The $\operatorname{Tr} A$ muscle thickness was determined by the distance between the superior border of the internal oblique muscle and inferior border of the $\operatorname{Tr} A$. This method for measuring $\operatorname{Tr} A$ is both reliable and noninvasive and has been used to successfully estimate $\operatorname{Tr} A$ activity (Bunce et al., 2002). Participants were instructed to inhale and hold their breath during US imaging because the $\operatorname{Tr} A$ thickness varies with the respiratory cycle (Ainscough-Potts, Morrissey, \& Critchley, 2006; Misuri et al., 1997). Measurements were performed under the following four conditions (Figure 1, 2, and 3):

- While the subject lay quiet in the prone position (a);

- While the subject bent over and touched the barbell on the floor with no intention of moving (b);

- While the subject stood, holding the barbell at knee level (c);

- While the subject stood, holding the barbell in the upright position (d).

Each position was held long enough for the examiner to obtain a clear US image of the TrA thickness. The participants held a barbell during the US measurements (conditions $\mathrm{c}$ and $\mathrm{d}$ ). The loard was prescribed individually for each subject as $20 \%$ of 1RM (repetition maximum).

\section{Reliability of measurements}

Data from five participants were chosen to examine both the intra-rater and inter-rater reliability of
US imaging. A single TrA thickness measurement was obtained from each subject with a 2-min interval between each measurements (total, five measurements). After each measurement, the participant stood up, walked around, and was then repositioned in the testing posture. The reliability across repeated measurements of the same image position was determined by intraclass correlation coefficients (ICC) (Rankin \& Stokes, 1998). The ICC for both inter- and intrarater reliability was greater than .95 for the $\operatorname{Tr} A$ muscle thickness measurements.

\section{Statistical analysis}

Statistical analyses were performed using Stastistica 5.1 (StatSoft, Tulsa, OK, USA). Analysis of variance (ANOVA) was used to examine similarities between the groups for age, height, weight, BMI, and maximum deadlift performance. Two-way ANOVA was performed for each condition (a-d), and was used to examine the significance between the groups and conditions. Tukey's honestly significant difference was used to account for multiple comparisons. Statistical significance was set at $p<.05$. The data are presented as means (standard deviation) unless otherwise stated.

\section{RESULTS}

We found no significant intergroup differences with respect to the participants' characteristics $(p>$ .05 ), including age, weight, height, and BMI (Table 1). Changes in $\operatorname{Tr} A$ muscle thickness was calculated between measurements obtained during each condition (b-d) with $\operatorname{Tr} A$ contracted and in the resting

TABLE 1

Participants'characteristics.

\begin{tabular}{|c|c|c|c|c|c|c|}
\hline & \multicolumn{2}{|c|}{ Elite $(n=8)$} & \multicolumn{2}{|c|}{ Control $(n=8)$} & \multirow{2}{*}{$t$} & \multirow{2}{*}{$p$} \\
\hline & $M$ & $S D$ & $M$ & $S D$ & & \\
\hline Age (year) & 39.9 & 8.8 & 34.6 & 7.0 & 1.31 & .21 \\
\hline Body height $(\mathrm{cm})$ & 169.1 & 8.8 & 168.9 & 6.5 & .05 & .96 \\
\hline Body weight (kg) & 83.4 & 27.3 & 74.4 & 10.3 & .87 & .40 \\
\hline $\mathrm{BMI}\left(\mathrm{kg} / \mathrm{m}^{2}\right)$ & 28.9 & 8.9 & 26.1 & 3.4 & .85 & .41 \\
\hline Raw Dedalift $(\mathrm{kg})^{*}$ & 245.3 & 47.8 & 185.9 & 35.9 & 2.81 & .01 \\
\hline Wilks equation & 177.9 & 26.0 & 133.7 & 17.2 & 4.01 & .00 \\
\hline
\end{tabular}

Legend: $\mathbf{n}$ - Number of participants; $\mathbf{M}$ - Mean; SD - Standard deviation; $\mathbf{t}$ - t-test; $\mathbf{p}$ - Probablity; BMI $\left(\mathrm{kg} / \mathrm{m}^{2}\right)$ - Bodi mass index; Raw Dedalift (kg) - Was performed in the classic style with the use of a lifting belt; Wilks equnition - Weight lifted by the powerlifter was multiplied by an index based on the body mass. 
(prone) position (a). The $\operatorname{Tr} A$ thickness measured during each contracted condition (b, c, and d) was divided by the $\operatorname{Tr} A$ thickness measured during the resting condition (a) to determine the relative change in TrA muscle thickness. For example, a thickness ratio of 2.0 indicates that the muscle thickness doubled from that during its resting position.

The interaction between groups and conditions was significant. TrA contractility was significant between conditions c (bar at the knee level) and d (bar at the top position) for both the elites and controls. In the elite group, there was a significant difference between conditions c (bar at knee level) and b (bar on the floor) ('Table 2).

Elite powerlifters demonstrated a $\operatorname{Tr} A$ contractile rate that was significantly greater than that for controls when the weight was at the knee level (c/a) (Table 2). However, there was no significant difference in the contractile rates when the bar was on the floor $(b / a)$ or in the top position $(\mathrm{d} / \mathrm{a})$.

\section{FIGURE 1}

Condition (b); The subject bent over and touched the barbell on the floor with no intention of moving.

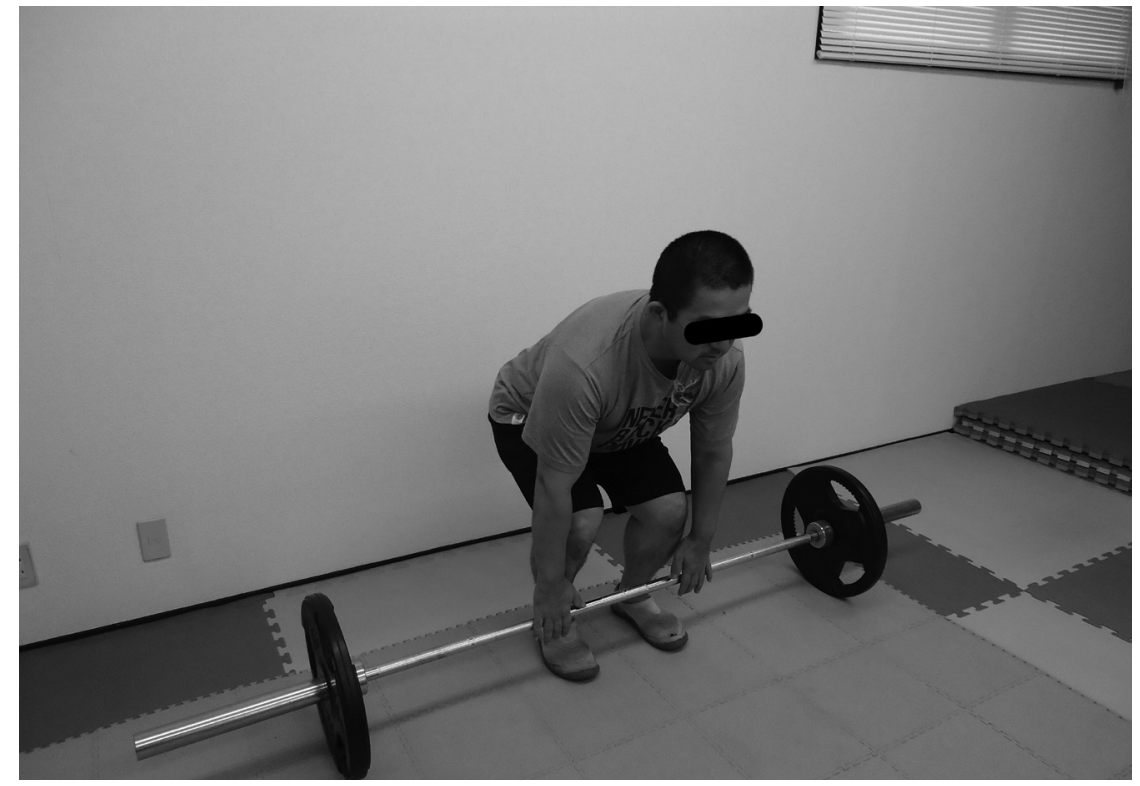

FIGURE 2

Condition (c); The subject stood, holding the barbell at knee level.

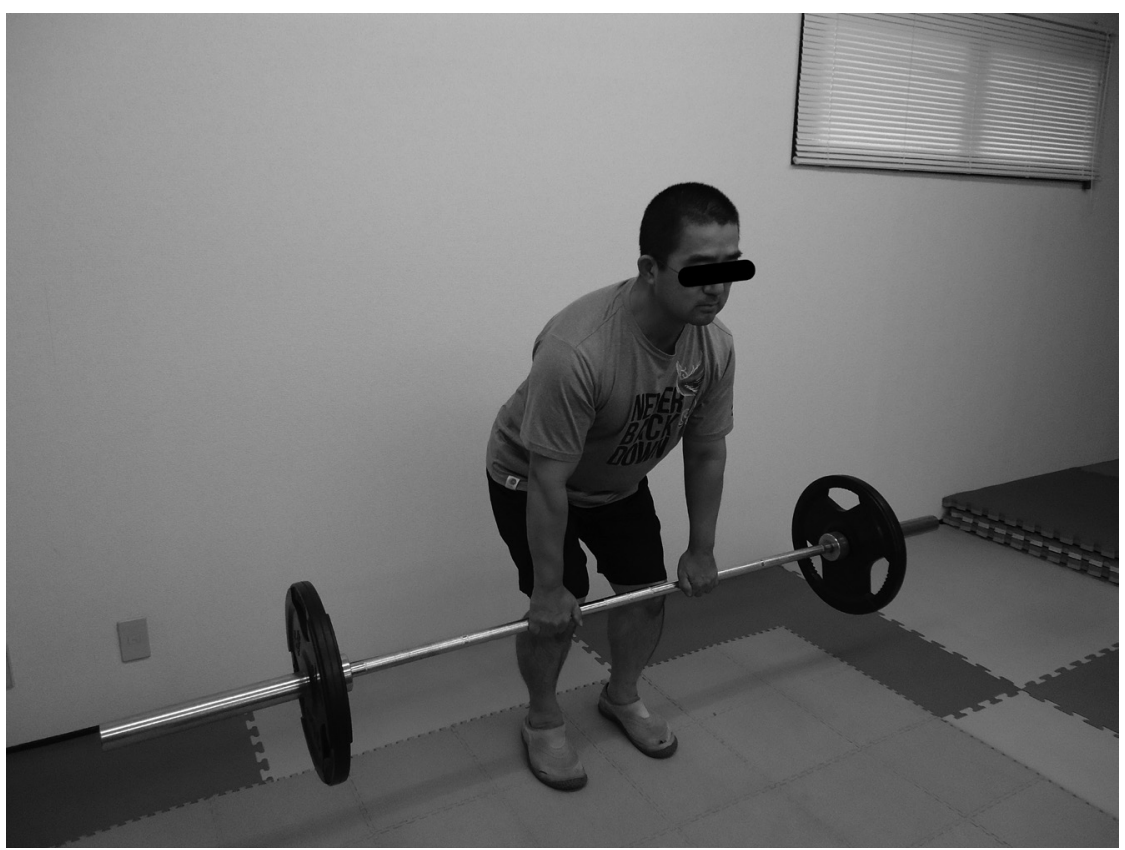


FIGURE 3

Condition (d); The subject stood, holding the barbell in the upright position.

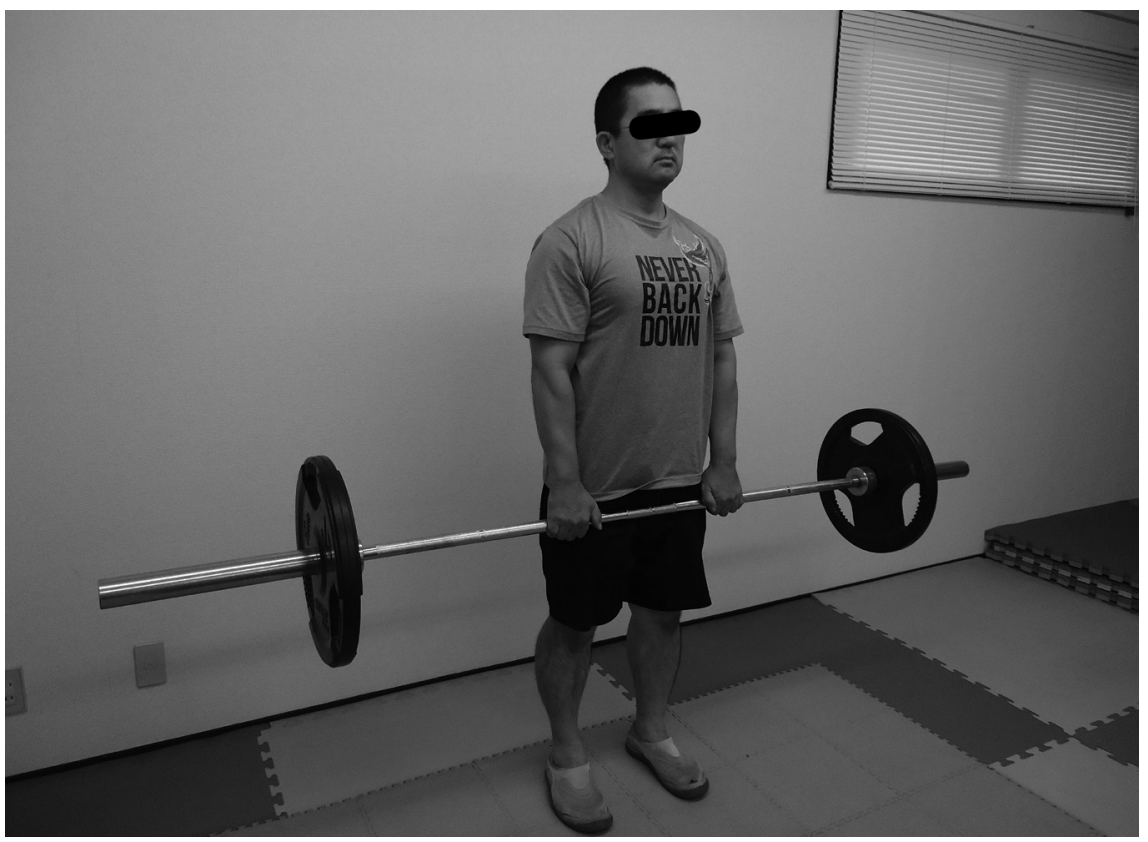

TABLE 2

Mean transversus abdominis (TrA) contractile rates during each condition.

\begin{tabular}{lcccccccccl}
\hline & \multicolumn{2}{c}{ Floor } & \multicolumn{2}{c}{ Knee } & \multicolumn{2}{c}{ Top } & \multicolumn{2}{c}{ Two-way ANOVA } & \multirow{2}{*}{ Post hoc. Turkey's HSD } \\
\cline { 1 - 7 } & $M$ & $S D$ & $M$ & $S D$ & $M$ & $S D$ & & \multicolumn{1}{c}{$F$} & \multicolumn{1}{c}{$p$} & \\
\hline Elite & 1.47 & .07 & 2.16 & .15 & 1.27 & .10 & F1 & 2.35 & .15 & Elite, Control: Knee > Top \\
\hline Control & 1.40 & .07 & 1.73 & .13 & 1.27 & .08 & F2 & 33.31 & $\mathbf{. 0 0}$ & Elite: Knee > Floor \\
\hline & & & & & & & F3 & 3.59 & .04 & Knee: Elite > Control \\
\hline
\end{tabular}

Thickness ratio $(M \pm S D)$ is the ratio of TrA thickness between the contracted and resting positions. A thickness ratio of 1.0 indicates no change in the TrA muscle thickness from the resting position.

Legend: M - Mean; SD - Standard deviation; F - F-test; $\mathbf{p}$ - Probablity; F1 - Gropus;

F2 - Conditions; F3 - Interaktions; Thickness ratio $(M \pm S D)$ is the ratio of TrA thickness between the contracted and resting positions. A thickness ratio of 1.0 indicates no change in the TrA muscle thickness from the resting position

\section{DISCUSSION}

Core stability is important for both strength and athletic performance. However, there is no direct evidence that establishes a relationship between core stability and athletic performance. Our study investigated whether core stability is related to deadlift performance in competitive powerlifters.

The relative $\operatorname{Tr} A$ contractility in the starting position did not differ between the groups. This may be because $\operatorname{Tr} A$ increases segmental stabilization and intra-abdominal pressure, and it activates in anticipation of loads to the spine. During the deadlift, the lumbopelvic area was exposed to maximum stress when the weight is at the knee level. Two previous studies reported that a sticking point, which is the weakest point in the range of motion, tends to occur around the knee, resulting from biomechanical disadvantages (Hales et al., 2009). Therefore, powerlifters should be able to generate high forces and have a stronger lumbopelvic region to overcome these biomechanical disadvantages. Based on our analysis, both groups showed higher TrA activity levels when the barbell was at the knee level. This result indicates that stronger lumbopelvic stability is indeed necessary at this point during movement. However, relative $\operatorname{Tr} A$ contractility was greater in elite powerlifters than in controls while the weight was held at knee level $(p=$ .04). Given that elite powerlifters showed higher $\operatorname{Tr} A$ contractile rates than controls when the barbell was at the knee level, the elite powerlifters showed greater lumbopelvic stability to clear the sticking point. 
This result indicates that deadlift performance can be influenced by $\operatorname{Tr}$ A function.

Relative $\operatorname{Tr}$ A contractility was not significant in elite powerlifters while holding the weight at the top position. This may be because holding the weight in the upright position not only depends on muscular contractility but also on the joint locking mechanisms.

It should be noted that a relatively small number of elite powerlifters were available for this study, which is a common situation in any study with elite athletes. Furthermore, only TrA was examined in this study. The contribution of other lumbopelvic stabilizers, such as lumbar multifidus, should be investigated in future studies.

Core strength training is often used with the aim to enhance athletic performance (McGill, 2001). However, few scientific studies have demonstrated a direct relationship between $\operatorname{Tr}$ A muscle contractile ability and athletic performance. The present findings suggest that deadlift performance can be influenced by $\operatorname{Tr}$ A function. Therefore, it is likely that core stability exercises along with weight training have the potential to increase the deadlift performance.

However further research should focus on the efficacy of neuromuscular training interventions that are aimed at improving core stability, and whether they can improve the deadlift performance.

\section{REFERENCES}

Ainscough-Potts, A. M., Morrissey, M. C., \& Critchley, D. (2006). The response of the transverse abdominis and internal oblique muscles to different postures. Manual Therapy, 11(1), 54-60. doi: 10.1016/j.math.2005.03.007; PMid: 16009592

Bergmark, A. (1989). Stability of the lumbar spine. A study in mechanical engineering. Acta Orthopaedica Scandinavica - Supplementum, 230, 1-54. doi: 10.3109/17453678909154177; PMid: 2658468

Bunce, S. M., Moore, A. P., \& Hough, A. D. (2002). M-mode ultrasound: A reliable measure of transversus abdominis thickness. Clinical Biomechanics, 17(4), 315-317. doi: 10.1016/ S0268-0033(02)00011-6

Cook, G. (2001). Baseline sports-fitness testing. In B. Foran (Ed.), High Performance Sports Conditioning (pp. 19-48). Champaign, IL: Human Kinetics Inc.

Cresswell, A. G., Grundstrom, H., \& Thorstensson, A. (1992). Observations on intra-abdominal pressure and patterns of abdominal intramuscular activity in man. Acta Physiologica
Scandinavica, 144, 409-418. doi: 10.1111/j.1748-1716.1992.tb09314.x; PMid: 1534959

Cresswell, A. G., Oddsson, L., \& Thorstensson, A. (1994). The influence of sudden perturbations on trunk muscle activity and intra-abdominal pressure while standing. Experimental Brain Research, 98(2), 336-341. doi: 10.1007/ BF00228421

Hales, M. E., Johnson, B. F., \& Johnson, J. T. (2009). Kinematic analysis of the powerlifting style squat and the conventional deadlift during competition: is there a cross-over effect between lifts? Journal of Strength \& Conditioning Research, 23(9), 2574-2580. doi: 10.1519/

JSC.0b013e3181bc1d2a; PMid: 19910816

Hodges, P. W. (1994). Is there a role for transversus abdominis in lumbo-pelvic stability? Spine, 4(2), 74-86.

Hodges, P. W., \& Richardson, C. A. (1996). Inefficient muscular stabilization of the lumbar spine associated with low back pain. A motor control evaluation of transversus abdominis. Spine, 21(22), 2640-2650. doi: 10.1097/ 00007632-199611150-00014: PMid: 8961451

Hodges, P. W., \& Richardson, C. A. (1997). Feedforward contraction of transversus abdominis is not influenced by the direction of arm movement. Experimental Brain Research, 114(2), 362-370. doi: 10.1007/PL00005644

Hodges, P. W., \& Richardson, C. A. (1998). Delayed postural contraction of transversus abdominis in low back pain associated with movement of the lower limb. Journal of Spinal Disorders, 11(1), 46-56. doi: 10.1097/00002517-19980200000008

Hodges, P. W., Richardson, C. A., \& Hasan, Z. (1997). Contraction of the abdominal muscles associated with movement of the lower limb. Physical Therapy, 77(2), 132-134. PMid: 9037214

Kanehisa, H., Ikegawa, S., \& Fukunaga, T. (1994). Comparison of muscle cross-sectional area and strength between untrained women and men. European Journal of Applied Physiology and Occupational Physiology, 148-154. doi: 10.1007/ BF00244028; doi: 10.1007/BF00843736

Kibler, W. B., Press, J., \& Sciasciam, A. (2006). The role of core stability in athletic function. Sports Medicine, 36(3), 189-198. doi: 10.2165/00007256-200636030-00001 Liemohn, W. P., Baumgartner, T. A., \& Gagnon, L. H. (2005). Measuring core stability. J Strength 
Cond Res, 19(3), 583-586. doi:

10.1519/1533-4287(2005)19[583:MCS]2.0.CO;2; doi: 10.1519/00124278-200508000-00016

Maughan, R. J., Watson, J. S., \& Weir, J. (1984). Muscle strength and cross-sectional area in man: a comparison of strength-trained and untrained subjects. Br J Sports Med, 18(3), 149-157. doi: 10.1136/bjsm.18.3.149: PMid: 6487941; PMCid: PMC1859378

McGill, S. (2001). Lower back stability: from formal description to issues for performance and rehabilitation. Exercise and Sport Sciences Reviews, 29(1), 26-31. doi: 10.1097/00003677200101000-00006

McGill S. (2002). Low back disorders. 2nd ed. Champaign, IL: Human Kinetics Inc.

McGill, S. (2010). Core training: evidence translating to better performance and injury prevention. Strength \& Conditioning Journal, 32(3), 33-46. doi: 10.1519/SSC.0b013e3181df4521

McGuigan, M. R. M., \& Wilson, B. D. (1996). Biomechanical analysis of the deadlift. Journal of Strength \& Conditioning Research, 10(4), 250-255. doi: 10.1519/00124278-199611000-00008; doi: 10.1519/1533-4287(1996)010<0250:BAOTD $>2$ .3.CO;2

Miller, M. I., \& Medeiros, J. M. (1987). Recruitment of internal oblique and transversus abdominis muscle during the eccentric phase of the curl-up exercise. Physical Therapy, 67(8), 1213-1217.

PMid: 2956614
Misuri, G., Colagrande, S., \& Gorini, M. (1997). In vivo ultrasound assessment of respiratory function of abdominal muscles in normal subjects. European Respiratory Journal, 10(12), 2861-2867. doi: 10.1183/09031936.97.10122861

Oddsson, L. I. (1990). Control of voluntary trunk movements in man: mechanisms for postural equilibrium during standing. Acta Physiologica Scandinavica Supplementum, 595, 1-60. PMid: 2080712

Panjabi, M. M. (1992). The stabilizing system of the spine. Part 1. function, dysfunction, adaptation and enhancement. Journal of Spinal Disorders, 5(4), 383-389. doi: 10.1097/00002517199212000-00001; PMid: 1490034

Parkhouse, K. L., \& Ball, N. (2011). Influence of dynamic versus static core exercises on performance in field based fitness tests. Journal of Bodywork and Movement Therapies, 15(4), 517-524. PMid: 21943626

Rankin, G., \& Stokes, M. (1998). Reliability of assessment tools in rehabilitation:an illustration of appropriate statistical analysis. Clinical Rehabilitation, 12(3), 187-199. doi: 10.1191/026921598672178340

Springer, B. A., \& Gill, N. W. (2007). Use of rehabilitative ultrasound imaging to characterize abdominal muscle structure and function in lower extremity amputees. Journal of Orthopaedic \& Sports Physical Therapy, 37(10), 635-643. doi: 10.2519/jospt.2007.2532; PMid: 17970411

Received: Sepetember 10, 2014 Revision received: December 11, 2014 Accepted: December 24, 2014 Correspondence to: Naoki Sakakibara, PhD The Department of Sports Medicine and Sports Science, Gifu University Schools of Medicine, Yanagido1-1, Gifu, Japan 501-1194 Phone: 0081582306421 Fax: 0081582306422 E-mail: drsakakibara@sportsdoc.jp 